\title{
Pitch Angle Properties of Magnetospheric Thermal Protons and Satellite Sheath Interference in Their Observation
}

\author{
J. J. SOJKA, ${ }^{1}$ G. L. WRENN, AND J. F. E. JOHNSON² \\ Mullard Space Science Laboratory, Holmbury St. Mary, Dorking, Surrey, England
}

\begin{abstract}
Cold proton observations from the geosynchronous GEOS 2 satellite are presented to show the pitch angle distribution of the refilling cold ion population found beyond the plasmapause. This refilling cold ion population flows into the depleted equatorial regions of the flux tubes from the topside ionosphere. This refilling is observed both day and night. The ions have energies between 0.5 and $3 \mathrm{eV}$. These energy characteristics are consistent with other recent observations. Although the observed angular distribution of these ions is highly complex, when allowance is made for the satellite sheath several conclusions can be made concerning the ion pitch angle distribution. The angular distribution is very field aligned with a characteristic source cone angle ranging from $10^{\circ}$ to $20^{\circ}$. This range of angles is consistent with ions originating below $20,000 \mathrm{~km}$ in the exosphere rather than in the heavy-ion ionosphere. As the flux tube density increases, the degree of field alignment decreases; however, based on the limited pitch angle coverage it is not possible to determine if the field-aligned component is simply being swamped by the local isotropic component or if the field-aligned component changes its pitch angle characteristics. A crucial aspect of the study was the availability of a unique data base of sheath-plasma observations. The observations were obtained by using the boom mounting and voltage biasing capabilities of the detector package in relation to the satellite. These data enabled us to describe how the satellite sheath affects the observation of cold $(<5 \mathrm{eV})$ protons. This qualitative understanding enabled the highly anisotropic data to be interpreted in terms of magnetospheric pitch angle distributions. The overall implications of this sheath-plasma coupling is that a technique to make satellite sheath observations has been demonstrated.
\end{abstract}

\section{INTRODUCTION}

Although the plasma transport processes controlling the refilling of the equatorial plasmasphere after magnetic storms have been much discussed over the past few decades, it is still only partially understood. Ionospheric thermal plasmas diffuse upward to the magnetic equator and undergo a series of interactions which trap them there. These mechanisms are not understood, but are generally referred to as pitch angle scattering, thermal plasma heating, and wave-particle interactions. Theoretical studies are not only restricted by the complexity of these processes but also by the almost total lack of measurements of these cold anisotropic plasmas (see Horwitz[1983] for a recent discussion of these problems). Recently, in situ plasma measurements have shown the temperature range and indicated some of the anisotropic characteristics of the filling ionospheric ion population [Olsen, 1982; Sojka et al., 1983; Wrenn et al., 1984]. These measurements are hampered by both instrumental limitations and unfavorable satellite sheath potentials. In the region where these measurements are made, $L>3$ near the magnetic equator, the ionospheric filling population occupies a source cone of $\left\langle 10^{\circ}\right.$ pitch angle. This severely restricts the interpretation of data from existing instruments, since they generally have angular acceptance

${ }^{1}$ Permanently at Center for Atmospheric and Space Sciences, Utah State University, Logan.

${ }^{2}$ Permanently at Department of Physics, University of Southampton, United Kingdom.

Copyright 1984 by the American Geophysical Union.

Paper number 4A8048.

0148-0227/84/004A-8048\$05.00 angles greater than $20^{\circ}$. However, it is precisely this measurement which is needed to identify the degree of pitch angle scattering which the ionospheric distribution has experienced in moving to the equator.

The problem of allowing for the satellite sheath potential is equally complex and is coupled to the interpretation of low-energy $E \lesssim 5 \mathrm{eV}$ plasmaspheric refilling data. All satellites in a plasma environment develop a sheath potential between them and the plasma. This potential in the geosynchronous environment can cause considerable problems for the operation of weather, communication, technological, and scientific satellites. Extensive studies have been carried out to increase our understanding of these problems. The review articles by Wrenn et al. [1979], Garrett [1981], and Whipple [1981] adequately describe our current understanding of satellite sheaths. The adverse effect the sheath electrostatic potential has upon the measurement of ambient plasma has long been known [Parker and Whipple, 1970; Whipple, 1976; Olsen, 1982]. These satellite sheath problems fall into two categories: (1) the satellite with a uniformly conducting surface, where a single well-behaved sheath forms, and (2) the satellite with different surface finishes, including insulators, where differential charging and large fluctuations in satellite potential occur. For this study, data from the geosynchronous GEOS 2 satellite are used. This satellite falls into category 1 and its potential relative to the plasma in the magnetosphere was typically $\sim+5 \mathrm{~V}$.

Although the GEOS 2 satellite has a well-behaved sheath, no in situ information on its sheath structure is available. In this paper we present refilling thermal proton data to show their pitch angle characteristics. Unfortunately, these protons have energies comparable to the typical sheath potential; hence these observations are integrally related to the effects of the satellite sheath. In 


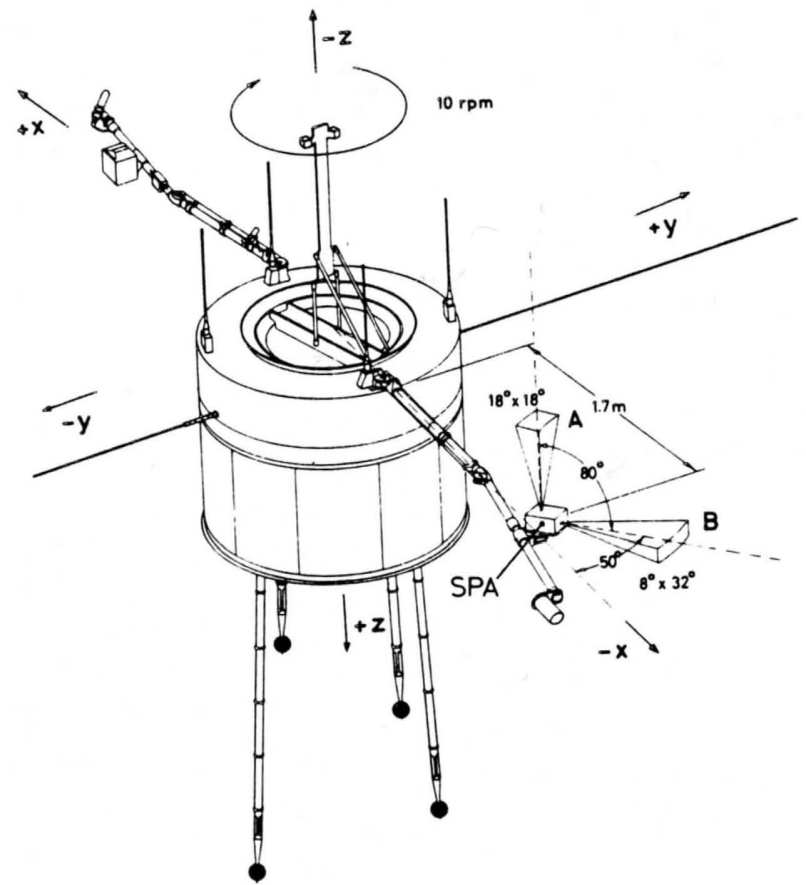

Fig. 1. Schematic of the SPA viewing apertures and boom mounting relative to the GEOS 2 satellite.

interpreting these data we present data and arguments to show that we qualitatively understand the effects the satellite sheath has upon our measurements.

\section{Suprathermal Plasma ANALyzer InStrument}

This paper uses the data acquired by the suprathermal plasma analyzers (SPA) flown on the geosynchronous GEOS 2 satellite. These analyzers, their operation, and the spacecraft environment are fully described by Wrenn et al. [1981]. Figure 1 shows the sensor location and the analyzer aperture geometry of the SPA instrument. The satellite is cylindrical with a radius of $0.8 \mathrm{~m}$. The SPA sensor package is boom mounted $1.7 \mathrm{~m}$ from the satellite body in such a way that it can be electrically biased with respect to the spacecraft "ground" potential. This has proved crucial to the measurement of thermal plasma beyond the plasmasphere. The GEOS 2 satellite is located at 6.6 $R_{E}$ close to the geographic equator and is oriented such that the spin axis is parallel to the earth's axis. In this configuration the local magnetic field is usually within $25^{\circ}$ of the satellite spin axis.

The SPA instrument consists of two hemispherical electrostatic differential energy analyzers. Each analyzer has an $11 \%$ energy resolution for positive ions and electrons within the 0.5 - to $500-\mathrm{eV}$ energy range. Figure 1 shows the viewing geometry for these two analyzers; aperture A is square and has an $18^{\circ} \times 18^{\circ}$ acceptance in a direction antiparallel to the satellite spin axis; aperture $B$ is rectangular with a $32^{\circ} \times 8^{\circ}$ acceptance as shown. These two orientations are such that analyzer A would view small pitch angles $\lesssim 25^{\circ}$, while analyzer $B$ would scan a range of large pitch angles $55^{\circ}$ to $105^{\circ}$. Under refilling conditions when thermal ions travel along the field line, analyzer A would be expected to observe larger fluxes than analyzer B.
The spin axis alignment of detector A was specifically selected to minimize spin modulation of the count rate and permit high time resolution of the measurements. An anisotropic flux would be expected to cause a small spin modulation of the detector $A$ count rate because the angular response of the analyzer is a function of both and $\beta$, the incident angles to the aperture normal measured in a plane through the center of the analyzer and the plane perpendicular, respectively. The $\alpha$ response is energy dependent, whereas the $\beta$ response is not [Wrenn et al., 1981]. During the GEOS 2 mission it was found that detector A often exhibited very large spin modulation at low energy. In the electron mode this mod. ulation is associated with the asymmetry of the photoelectron cloud around the spacecraft, with the minimum count rate corresponding to the orientation of the sensor antisunward of the spacecraft. In the proton mode the modulation was observed to be more complex, and a study of this effect leads to important conclusions concerning the influence of the satellite sheath on the observed refilling thermal proton fluxes.

\section{Satellite Sheath Modulation OF THERMAL ION FLUXES}

\subsection{Detector A Spin Anisotropy}

In this section the spin modulation observed in detector A when GEOS 2 was in the magnetosphere is studied in order to understand the satellite sheath effects upon the observed thermal ion fluxes. Detector A is used because its aperture is parallel to the satellite spin axis and hence views the same region of the magnetospheric plasma phase space during a spin period, resulting in a constant count rate with spin angle assuming no major temporal or spatial changes over a 6-s spin period. Data are presented for a summer and winter day of moderate activity as well as for a very quiet day. These two moderately active days are representative of about $90 \%$ of all the data observed by GEOS 2 in a 2-year period. The quiet day is typical of the few percent of days when GEOS 2 remained in the plasmasphere for a whole day. The remaining days were extremely active.

Various detector A spin-peak count rate curves are shown in Figure 2. For particular UT's from the selected 3 days of data the peak count rates were obtained from the individual low-energy differential count rate spectra. These proton spectra all showed a narrow peak at an energy corresponding to the total acceleration the magnetospheric thermal ions had experienced in passing through the satellite sheath and detector bias into the A detector. Curves 1 and 2 are both from the quiet highdensity day (March 12, 1980). During the period when the satellite was in eclipse (2130 UT), no significant spin modulation is observed. Curve 2 at $1230 \mathrm{UT}$ is typical of the modulation associated with the solar cell array problem. This curve shows that no spin variation occurs between $90^{\circ}$ and $275^{\circ}$ and that at $65^{\circ}$ and $300^{\circ}$ the peak count has dropped to half the maximum plateau value. For spin angles near $0^{\circ}$ the peak count rate is an order of magnitude lower than the maximum value. A detailed discussion of the solar cell array problem is given below. Curves 3 and 4 correspond to the spin modulation at 1825 
GEOS-2, SPA Cold Proton, Detector A

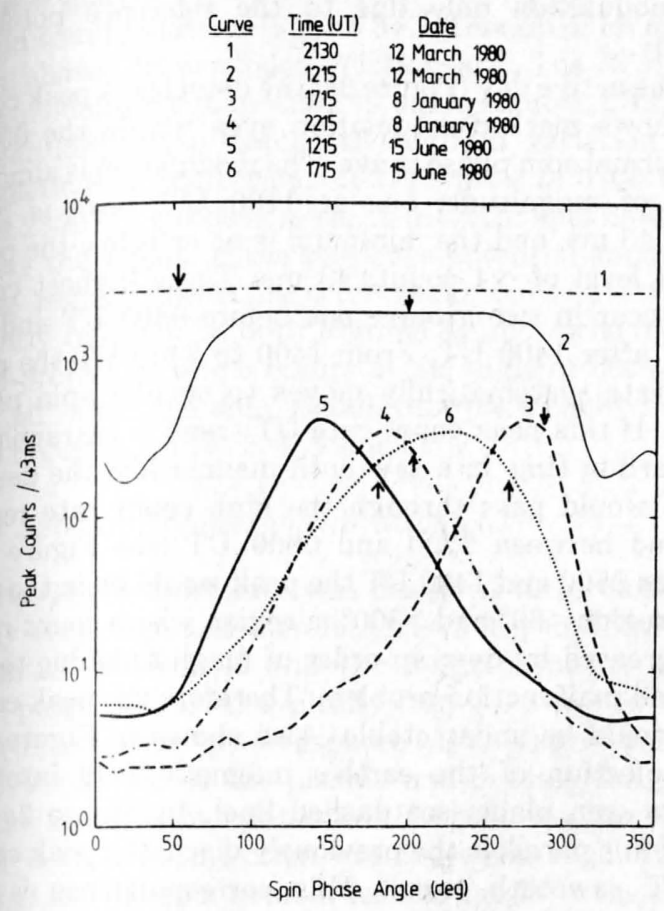

Fig. 2. Spin phase modulation of the cold proton peak count rate at selected UT's for days 8,72 , and 167,1980 .

and 2225 UT, respectively, on January 8, 1980. Comparing these two curves shows that whereas curve 4 is symmetric about its peak, curve 3 is skewed with a sharper falloff at large spin phase angles. This sharper falloff is associated with the solar cell array problem; i.e., compare with falloff in curve 2 . The width of curve 4 at half the

GEOS-2, SPA Detector A

1980 day 8

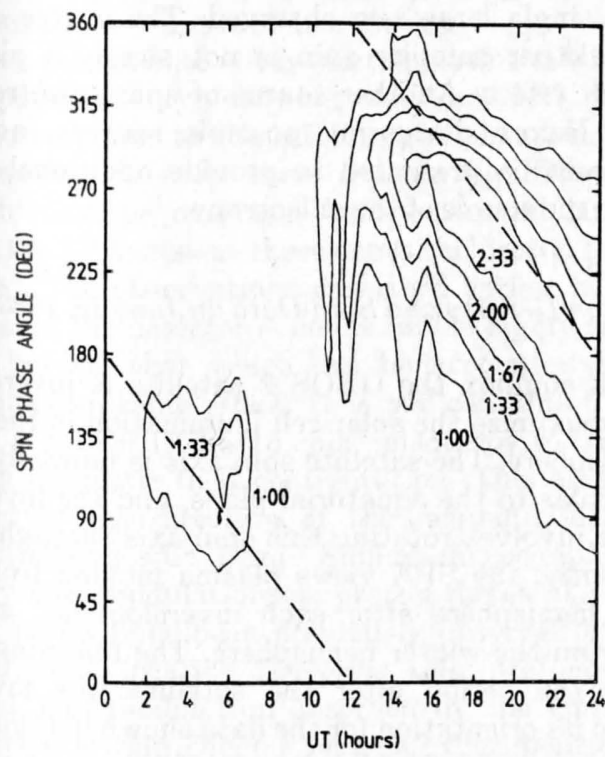

Fig. 3a. Logarithmic contours of detector A cold proton peak count as a function of UT and satellite spin phase angle for day 8,1980 . Contours are labeled at 0.33 intervals of logarithmic to base 10 of the count rate $/ 43 \mathrm{~ms}$. The dashed lines represent the spin phase of the magnetic field projected into the satellite spin plane as a function of UT.
GEOS-2, SPA Detector A 1980 day 72

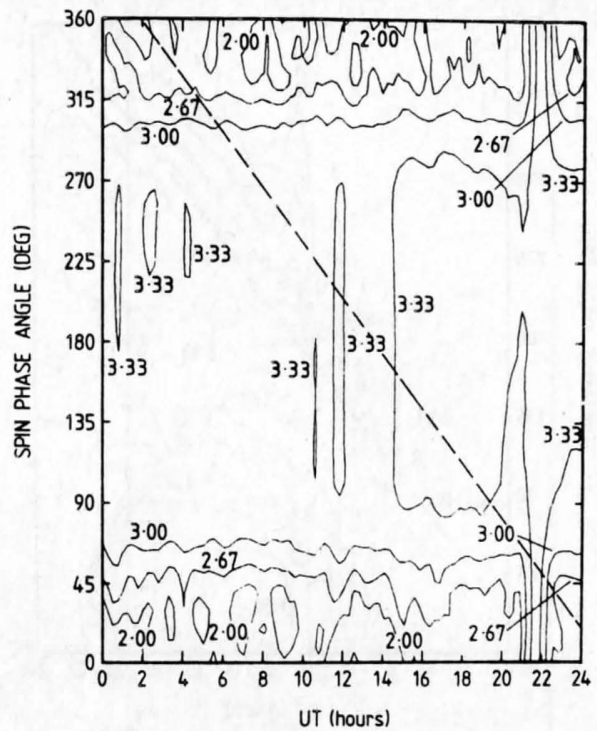

Fig. $3 b$. Same as Figure $3 a$ except for day 72, 1980.

maximum peak count rate is $\sim 60^{\circ}$. Data from June 15 , 1980 , at 1215 and 1825 UT are plotted as curves 5 and 6 , respectively. Again skewing is present near low and at high spin angles; however, the curves are somewhat different from the previous two. Curve 6 has a very broad half width, approximately $105^{\circ}$, compared to the $60^{\circ}$ for curve 4. Also shown on these curves in Figure 2 is the corresponding spin phase angle of the magnetic field projection into the satellite spin plane. For these data and the other data presented in this paper the magnetic field direction varies from a few degrees to $\sim 30^{\circ}$ of the satellite spin axis direction. For cuves $3,4,5$, and 6 this projected angle follows the maximum peak count by about $40^{\circ}$. Curve 2 shows that the modulation at $60^{\circ}$ and again at $300^{\circ}$ associated with the satellite solar cell malfunction is not sharply defined. It affects count rates within the $60^{\circ}$ to $300^{\circ}$ spin phase range; only count rates between $100^{\circ}$ and $250^{\circ}$ are unaffected. All the detector A spin modulations shown in Figure 2 are characteristic repeatable observations. Since no such modulation is expected at a fixed phase space location in the source plasma, these modulations are associated with satellite instrument viewing problems.

\subsection{Proton Spin Modulation on January 8 and March 12, 1980}

Figure 3 shows contours of the detector A thermal proton peak count rate/43 ms as a function of UT and satellite spin phase angle. This parameter has been chosen because it is easily identifiable and has the lowest statistical errors. The logarithm of the peak count rate to the base 10 is contoured at intervals of 0.33 . Figure $3 a$ is for January 8, 1980 (day 8), and Figure $3 b$ is for March 12, 1980 (day 72), the same two periods used for Figure 2. The spin phase angle is measured from the direction at which detector B looks at the sun and is defined in the opposite sense to the actual satellite rotation angle in order to obtain a right-handed coordinate system for the SPA 
GEOS-2, SPA Detector A 1980 day 167

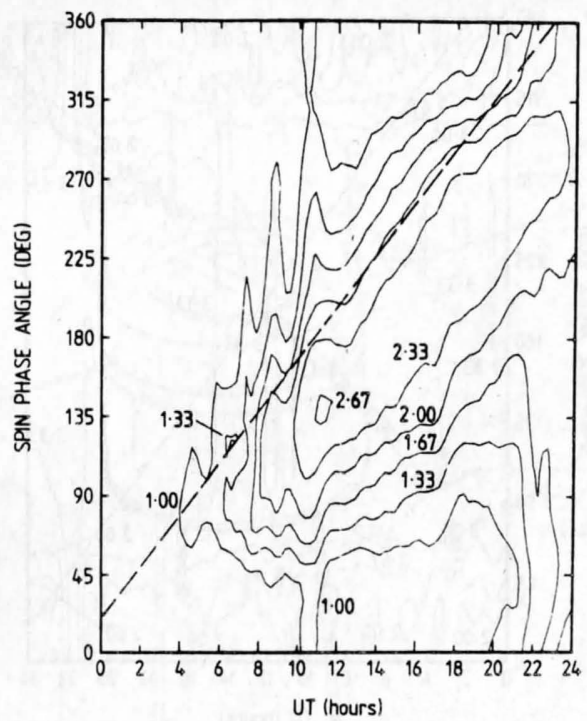

Fig. 4. Logarithmic contours of detector A cold proton peak count rate as a function of UT and satellite spin phase angle on day 167,1980 . The contouring layout is identical to Figure 3.

package (note that the SPA boom aligns with the sun at a phase angle of $50^{\circ}$; see Figure 1).

Both Figures $3 a$ and $3 b$ show marked, but quite distinct, spin modulation. During the refilling day (Figure $3 a$ ) the spin modulation changes phase with UT. On the isotropic day (Figure $3 b$ ) the modulation is UT independent. This latter modulation is discussed first, since it is a consequence of a solar cell array malfunction [Wrenn, 1978]. As the satellite spins, different sections of the solar cell array on the cylindrical body come into sunlight. Unfortunately, one string of cells developed a short circuit to the satellite structure on August 5, 1978 [Kalweit, 1979]. Consequently, for $1 / 3$ of each satellite spin period when the faulty solar cell string is illuminated, the central ground point (CGP) of the spacecraft is raised by $12.5 \mathrm{~V}$. The SPA instrument references its bias voltages in relation to this CGP. This effectively increases the sensor bias with respect to structure by the $12.5 \mathrm{~V}$ and partially negates the effect of the applied negative bias. Thermal ions during this $1 / 3$ spin period are accelerated by a smaller voltage; and consequently, ion fluxes to both detectors $\mathrm{A}$ and $\mathrm{B}$ decrease. In Figure $3 b$ the peak count rates between $60^{\circ}$ and $300^{\circ}$ are approximately constant at about 1000 counts $/ 43 \mathrm{~ms}$. This is the normal configuration. At angles below $\sim 60^{\circ}$ and greater than $\sim 300^{\circ}$ the peak count rate decreases to below 100 counts $/ 43 \mathrm{~ms}$ due to the artificially changed SPA bias voltage. More extensive discussions on the effects of this changing bias voltage are given by Wrenn et al. [1979] and Johnson [1981]. At 2130 UT on March 12, 1980 , the satellite goes into eclipse, the solar cells become nonoperational, and the changing bias voltage disappears. Figure $3 b$ shows how at 2130 UT the peak count rate has become constant with spin phase angle. The general effect of this satellite malfunction is to reduce the peak count rates by over an order of magnitude below $60^{\circ}$ and above $300^{\circ}$ spin phase angle. On the quiet day, where the density was $\sim 80 \mathrm{~cm}^{-3}$ and the cold protons were isotropic, the detector A count rate shows significant spin modulation only due to the reference potential anomaly.

On the active day (Figure $3 a$ ) the detector A peak count rate shows marked modulation even within the $60^{\circ}$ to $300^{\circ}$ normal spin phase range. The modulation is almost 2 orders of magnitude; i.e., at $1700 \mathrm{UT}$, peak is $>200$ counts $/ 43 \mathrm{~ms}$, and the minimum is at or below the background level of $\sim 4$ counts $/ 43 \mathrm{~ms}$. These highest count rates occur in two groups: one before 0800 UT and the second after 1400 UT. From 1400 to 2400 UT the peak count rate systematically moves to smaller spin phase angles. If this peak count rate-UT trend is extrapolated backward in time in a sawtooth manner like the dashed line, it would pass through the high count rate region observed between 0200 and 0800 UT (see Figure $3 a$ ). Between 0800 and 1400 UT the peak would lie in the spin phase region $<60^{\circ}$ and $>300^{\circ}$, a region where count rates are decreased by over an order of magnitude due to the solar cell malfunction problem. Therefore the peak count rates would be undetectable. Also shown in Figure 3 is the projection of the earth's magnetic field into the satellite spin plane (see dashed line). In Figure $3 a$ the dashed line parallels the previously discussed peak count rate-UT sawtooth trend. This correspondence is not unique to day 8, 1980; in fact, all winter disturbed days show the same trends when refilling is present.

The spin modulation on detector $\mathrm{A}$ is quite unexpected even given that the pitch angle distribution is highly anisotropic. During a 24-hour UT period, detector A's central pitch angle varies slowly between $12^{\circ}$ and $25^{\circ}$. This modulation is attributed to the local time dependent geometry of the geosynchronous magnetic field line. Because of the relatively large acceptance angle of detector A $\left( \pm 9^{\circ}\right)$, one expects that detector spin anisotropies associated with the square aperture geometry or the $\alpha, \beta$ aperture angles could give rise to four or two small count rate peaks per satellite spin rather than the single large one observed. The source of the single peak per satellite spin is not simply a plasmainstrument effect. Another source of spin anisotropy is required. Before discussing possible sources, another data set will be presented to provide additional information on the source of the anisotropy.

\subsection{Proton Density and Spin Data on June 15, 1980}

At each equinox the GEOS 2 satellite is inverted in order to maximize the solar cell illumination in the summer hemisphere. The satellite spin axis is normally kept perpendicular to the equatorial plane, and the inversion maneuver involves rotating this spin axis through $180^{\circ}$. Consequently, the SPA views plasma moving from the opposite hemisphere after each inversion, i.e., always coming from the winter hemisphere. The following data are from the period after the satellite was inverted relative to its orientation for the data shown in Figure $3 a$. Figure 4 shows logarithmic contours of detector A proton peak count rate as a function of UT and spin phase angle for the inverted day, June 15, 1980. Also shown is the UT spin phase variation of the magnetic field projection in the satellite spin plane (dashed line). Again, the A detec- 
tor sees marked spin modulation which is somewhat similar in trend to that in Figure $3 a$, although of an opposite spin phase-UT sawtooth relationship. The A detector spin modulation amplitude is again almost 2 orders of magnitude,-and it tracks the diurnal variation of the magnetic field projection. At UT's prior to 0700 UT and after 2300 UT the peak in the A detector spin modulation enters the region of the reference potential anomaly. In these regions the A detector peak count rate is significantly reduced. In both Figures $3 a$ and 4 the magnetic field projection angle occurs at an angle of between $30^{\circ}$ and $50^{\circ}$ after the spin modulation peak count rate (see also Figure 2, curves $3,4,5$, and 6 ).

\subsection{Satellite Sheath Considerations}

The GEOS 2 satellite was designed to be electrostatically very clean and therefore has a very uniform sheath potential between it and the magnetospheric plasma. This potential varied typically from -0.5 to $+10 \mathrm{~V}$, such that in the denser plasmasphere the satellite would be at $-0.5 \mathrm{~V}$ relative to the plasma and outside the plasmasphere at a positive potential. Even during the severest geosynchronous injection events, when high fluxes of tens of $\mathrm{keV}$ plasma bombarded the satellite, the satellite potential remained below $+20 \mathrm{~V}$. When the satellite is positive, thermal protons are unable to overcome the sheath potential to reach the SPA detectors. To help these protons reach the SPA, a negative bias is applied to bias the SPA relative to the rest of the satellite. This potential was $-28 \mathrm{~V}$ for the data shown in Figures 2, 3, and 4. Under relatively dense plasmaspheric conditions when the satellite sheath potential is approximately zero, the $-28-\mathrm{V}$ bias accelerates protons very effectively into the SPA (see earlier discussion). The inferred density is consistent with that observed by other GEOS 2 instruments [Decreau et al., 1978]. Indeed, the bias voltage causes no significant spin angle anisotropies or other distortions to the count rate distribution.

When the satellite is beyond the plasmasphere, the satellite potential is typically between 5 and $10 \mathrm{~V}$. This situation corresponds to the refilling periods associated with Figures $3 a$ and 4 . At these times a significant satellite sheath must be overcome before thermal protons can reach the SPA; also at these times the proton flux is field aligned. The observations described earlier in section 3 show that the detector A count rate is highly spin modulated beyond that which can be accounted for by an instrument-plasma effect. It is our contention that this effect is primarily due to spin anisotropy in the satellite sheath magnetic field configuration. This anisotropy is related to the direction of the magnetic field in the satellite-boom spin plane. Similar large-amplitude lowenergy spin modulations of proton fluxes at very small pitch angles have been previously observed at the geosynchronous location [Deforest and McIlwain, 1971]. Indeed, this modulation observed by the University of California at San Diego's plasma experiment on ATS 5 was confirmed to be caused by differential charging effects associated with the ATS 5 satellite sheath environment [Deforest, 1973].

Our understanding of satellite sheaths is still incomplete; however, the basic physical phenomena governing

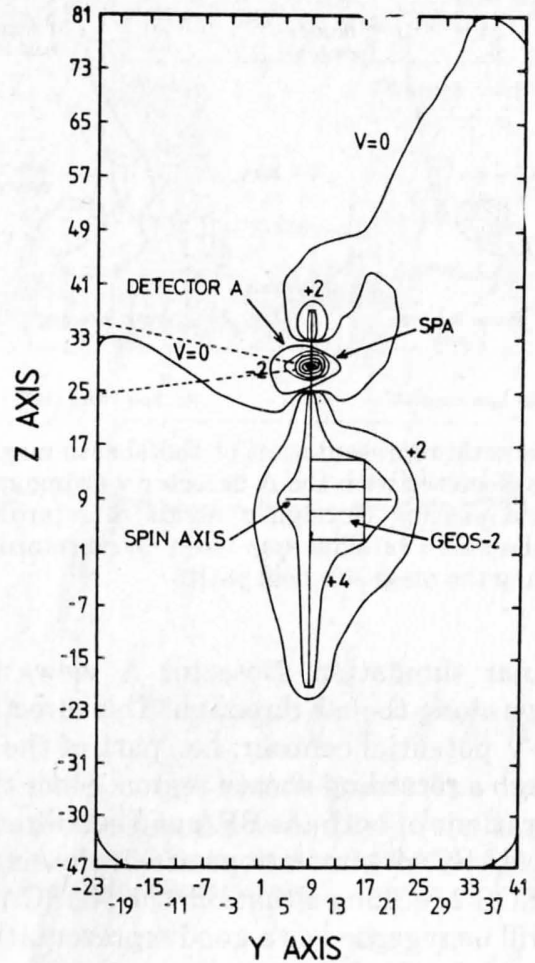

Fig. 5. Cross section of GEOS 2 satellite sheath potentials taken in the SPA boom-spin axis plane. The contours are drawn at 2-V intervals and represent the NASCAP model sheath solution for a satellite potential of $5 \mathrm{~V}$ and an SPA package potential of $-28 \mathrm{~V}$. The $Y$ and $Z$ axes are labeled in units equal to the grid cell size used in the simulation.

them are understood [Garrett, 1981; Whipple, 1981; Sagalyn et al., 1983]. For satellites with complex geometries, i.e., booms, antenna, and other surface projections, no analytic description of the sheath potential is available. However, numerical models which study or simulate sheaths have been developed with some success in regions where the plasma Debye length is comparable to or larger than the satellite dimensions. This criterion is met for the GEOS 2 satellite operating beyond the plasmasphere, the region of refilling flux tubes.

One such computer model extensively used to simulate satellite sheaths in the geosynchronous environment is the NASCAP model described in detail by Mandell et al. [1978]. This computer model solves LaPlace's equation and hence does not allow for space charge effects, a possibly serious omission. However, the NASCAP model satellite potential predictions have been successfully compared with geosynchronous satellite observations by Olsen et al. [1981]. Figure 5 is a NASCAP model potential calculation for the GEOS 2 sheath for the SPA -28-V bias configuration described above [Katz et al., 1978]. This figure is a schematic simplification of a more detailed figure by Katz et al. [1978], with the detector A aperture geometry being indicated by dashed lines. Contours of potential are drawn at $2-\mathrm{V}$ intervals in a cross section through the SPA boom and the GEOS 2 spin axis. The satellite and boom are at $+5 \mathrm{~V}$ relative to the plasma boundary; the SPA is at $-28 \mathrm{~V}$ relative to the satellite. The coordinate system represents the computer model grid cell size and therefore indicates the best resolution of 


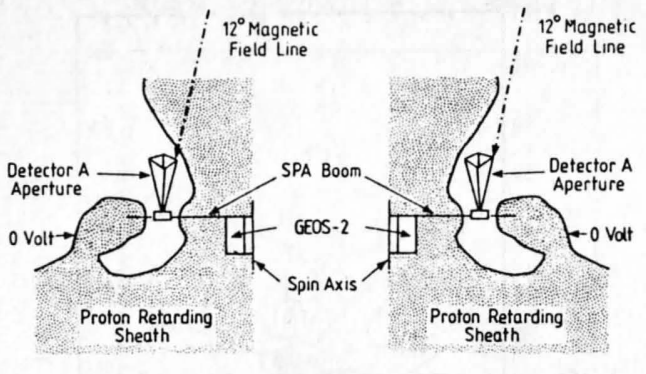

(a) Spin Phase $=0^{\circ}$

(b) Spin Phase $=180^{\circ}$

Fig. 6. Schematic representation of the sheath-magnetic field asymmetry associated with the A detector viewing aperture. (a) Magnetic field passing through a region of retarding sheath potential while half a satellite spin later, $(b)$ no retarding region is present along the magnetic field path.

this particular simulation. Detector A views from the SPA package along the $-Y$ direction. This direction intersects the $\mathrm{O}-\mathrm{V}$ potential contour; i.e., part of the aperture views through a retarding sheath region. Since the potential configurations of both the SPA and satellite potential used in the NASCAP simulations are in close agreement with the GEOS 2 refilling situation, the NASCAP sheath geometry will be regarded as a good representation of the GEOS 2 sheath for the following discussions.

Figure 6 shows the problem of collecting protons through a retarding (+ve) sheath for two satellite spin phases separated by half a spin. Each panel shows the same SPA boom spin axis cross section with only the Figure 5 NASCAP O-V potential contour being drawn. The shaded area indicates regions of positive potential relative to the plasma; i.e., cold ions entering these regions are retarded. In Figure $6 a$ the magnetic field, tilted at $12^{\circ}$ to the A detector aperture normal, passes through the retarding region of the sheath. Half a satellite spin later (Figure $6 b$ ) the same field line can reach the aperture without passing through the retarding region. Contrasting the flow of field-aligned cold protons into detector $\mathrm{A}$ for Figures $6 a$ and $6 b$ reveals two quite different situations. When GEOS 2 is outside the plasmasphere, the cold proton trajectories are approximately straight lines in the sheath interaction region. Table 1 compares the plasma Debye length $\lambda_{D}$ with the proton Larmor radius $L_{r}$ for typical plasma conditions. The Larmor radius is $10-100$ times larger than the Debye length. Since the Debye length is a characteristic dimension of the sheath, we can conclude that protons with small pitch angles traverse the sheath in much less than a gyration, i.e., in a straight line. As these protons traverse the sheath they do, however, experience electric fields. These fields will modify the trajectories and in some cases reflect low-energy ions away from the satellite. In Figure $6 a$ the field-aligned protons would experience such deflecting or even reflecting electric fields. No such reflecting fields are present for field-aligned protons in Figure $6 b$. Therefore the count rate for the Figure $6 b$ configuration would be expected to exceed that of Figure $6 a$ if the plasma were highly field aligned. Indeed, it is this very effect, namely, the retarding sheath electric fields, which has prevented previous satellite experiments from viewing these cold magnetospheric protons [Olsen, 1982].

In Figures 5 and 6 a two-dimensional view of how the sheath equipotentials are orientated relative to the detector A aperture is shown. In the third dimension, looking down onto the aperture, the satellite geometry is also highly asymmetric. Figure 7 shows a plan view of the GEOS 2 satellite, SPA boom and SPA package. The boom and satellite are to scale, but for reasons of clarity the SPA package has been enlarged to enable the aperture geometry to be seen. Also shown in Figure 7 is the definition of the SPA spin phase. It is taken from the B detector viewing direction projected into the spin plane and increases as shown. Thus zero-degree spin phase occurs when detector B views directly at the sun. Referring back to Figures $3 a$ and 4 the detector A peak count rate occurs $30^{\circ}$ to $50^{\circ}$ of spin phase before the magnetic field line projection into the spin plane. In terms of Figure 7 the detector A peak count rates occur when the magnetic field projection through the SPA lies within the shaded sector of Figure 7. This shaded region of satellite spin phase angles would also correspond to the magnetic field sheath example shown in Figure $6 b$, for which the count rates were expected to be large. In section 3.1 the examples of the detector A spin modulation showed that the peak covered a relatively wide range of phase angles from $\pm 30^{\circ}$ to $\pm 50^{\circ}$. Therefore for almost a quarter of each spin while the magnetic field projection lies near and within the shaded cone in Figure 7, detector A would experience high count rates. This whole region represents trajectory geometries which do not need to cross the main satellite sheath to reach the detector A aperture. At other spin angles the proton trajectories would have to intersect the repulsive electric fields associated with the satellite sheath.

The above qualitative description of the sheath-mag. netic field spin phase anisotropy is highly suggestive of the source of the detector A count rate spin modulations observed during field-aligned refilling periods. Quantitatively, the details of how the sheath geometry would be modified in a magnetized plasma are largely undetermined. Consequently, the zeroth-order sheath potentials shown in Figures 5 and 6 cannot be used with confidence

TABLE 1. Characteristic GEOS 2 Debye Lengths and Larmor Radii

\begin{tabular}{lrcccr}
\hline \multicolumn{1}{c}{ Location } & $n, \mathrm{~cm}^{-3}$ & $T_{e} \cong T_{\text {proton }}, \mathrm{eV}$ & $\lambda_{D}, \mathrm{~m}$ & $L_{r}^{*}$ at $5^{\circ}$ Pitch Angle, $\mathrm{m}$ & $L_{r}^{*}$ at $30^{\circ}$ Pitch Angle, $\mathrm{m}$ \\
\hline $\begin{array}{l}\text { Plasmasphere } \\
\text { Plasmapause }\end{array}$ & 1000 & 0.6 & 0.18 & 97. & 556. \\
$\begin{array}{l}\text { Refilling region beyond } \\
\text { plasmasphere }\end{array}$ & 10 & 1.5 & 2.8 & 153. & 880. \\
\hline
\end{tabular}

*Computed assuming magnetic field of $100 \gamma$. 
for a more comprehensive study. The detector A count rate is not simply dependent on field-aligned protons; protons of pitch angles over a wide range can enter the aperture (see Figure 1). Therefore proton trajectories would have to be traced backward from the detector aperture through the sheath to determine their original plasma pitch angles, and consequently, their contribution to the total count rate. This procedure would, given the high degree of uncertainty in both the sheath configuration and proton pitch angle distribution, lead to questionable results. Hence for the remainder of this study only the qualitative aspects of the sheath effect will be considered.

\section{Thermal Proton Oservations}

At the GEOS 2 geosynchronous location of $6.6 R_{E}$ on the geographic equator the magnetic flux tubes are usually in a depleted state and are therefore subject to refilling by thermal plasma flowing up from lower altitudes. This thermal plasma has a proton component of 0.5-4 eV temperature and is field aligned [Sojka et al., 1983; Wrenn et al., 1984]. Less frequently, when the equatorial density exceeds $\sim 10 \mathrm{~cm}^{-3}$, the character of the thermal proton component changes. It becomes cooler $(<1 \mathrm{eV})$ and almost isotropic in pitch angle; this is used to identify regions of plasmaspheric plasma.

The SPA was regularly operated in a cold proton mode which was ideal for monitoring the state of filling of the GEOS 2 flux tube. In this mode a sensor bias of $-28 \mathrm{~V}$ accelerates any cold ions through the positive spacecraft potential into the analyzer. The count rates for the eight energy channels above the effective accelerating potential are integrated to calculate the number density of these ions. The integrations are split into 16 spin phase bins. The seven antisunward bins with the maximum count rates are used for the density calculation; this restriction in spin phase angle is due to the solar cell array potential problem afflicting GEOS 2 [Johnson, 1981]. Count rate accumulations are made over 6-min periods, but generally the experiment would only be in a suitable mode for a small fraction of this time. This results in incomplete coverage of the spin phase bins and is the reason for selecting only seven out of a possibly $\sim 10$ useful bins. It is assumed that the majority of the ions are protons, and their temperature is less than $5 \mathrm{eV}$.

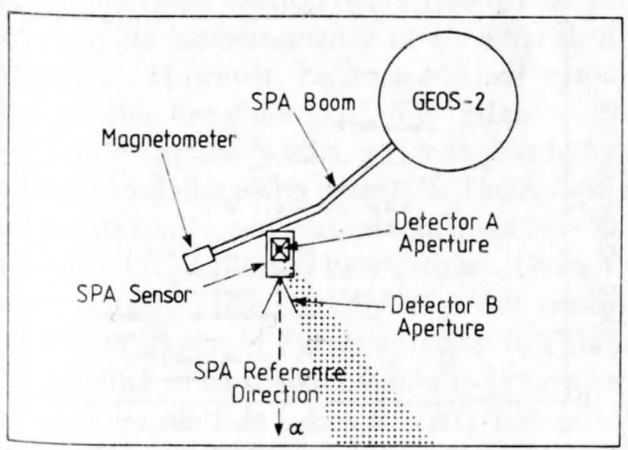

Fig. 7. Plan view of the GEOS 2 satellite showing detector A aperture, SPA boom, and satellite geometry. The shaded area represents the spin phase location of the magnetic field projection at the time detector A's count rate is maximized.
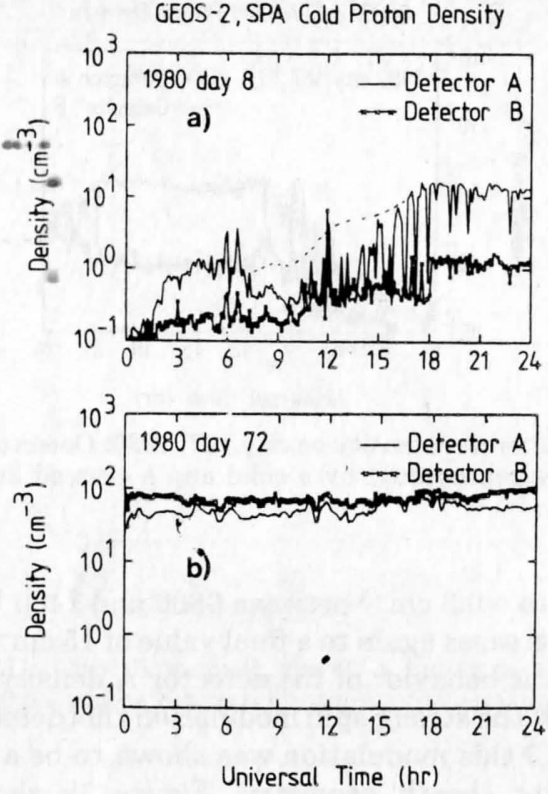

Fig. 8. Cold proton densities on $(a)$ day 8,1980 , and $(b)$ day 72 , 1980. Detector A and B densities are represented by a solid line and a crossed solid line respectively.

Therefore in this automated procedure we compute the first moment of the distribution function over a selected region of phase space to obtain the proton density. This program also calculates the second moment of the distribution function (temperature); however, no temperature data will be presented in this study. These two moments are in good agreement with density anv temperature inferred from the analysis of individual energy spectra and data obtained by other instruments on GEOS 1 and 2 [Decreau et al., 1978].

\subsection{Proton Densities on January 8 and March 12, 1980}

Figure 8 shows two 24-hour density profiles produced from the above analysis of cold proton mode data, illustrating the field-aligned refilling state (Figure $8 a$ ) and the isotropic plasmasphere situation (Figure $8 b$ ). March 12, 1980 (day 72, Figure $8 b$ ), was geomagnetically quiet $(\Sigma K p$ $=2^{+}$). During this period the flux tube is within the plasmasphere for the whole day (see earlier definition of plasmasphere). The densities are high $\left(50-80 \mathrm{~cm}^{-3}\right)$ the spacecraft potential is low ( 0.5 V) [Knott et al., 1984]; and the distribution is nearly isotropic with a temperature of $0.7 \mathrm{eV}$. The higher density values for detector $\mathrm{B}$ are explained by either a departure from corotation or a small pitch angle anisotropy of the plasma [Wrenn et al., 1984].

Figure $8 a$ shows proton density data for January 8 , 1980 (day 8). This is a slightly more disturbed day with a $K p$ sum of 12 . The days prior to this one were also disturbed, and during this whole period GEOS 2 was outside the plasmasphere. Over the 24-hour period the detector B density increases from less than $0.1 \mathrm{~cm}^{-3}$ to 1.5 $\mathrm{cm}^{-3}$ while detector A density, although larger, is considerably more erratic. Detector A density increases from $0.1 \mathrm{~cm}^{-3}$ to a value of $1.5 \mathrm{~cm}^{-3}$ by $0400 \mathrm{UT}$, but then 


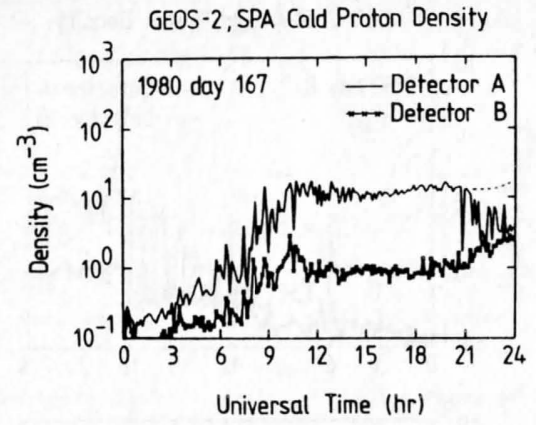

Fig. 9. Cold proton density on day 167, 1980. Detector A and B densities are represented by a solid and a crossed line, respectively.

decreases to $\sim 0.3 \mathrm{~cm}^{-3}$ between 0800 and $1400 \mathrm{UT}$, after which it increases again to a final value of $15 \mathrm{~cm}^{-3}$.

The erratic behavior of the detector A density is a consequence of the strong spin modulation this detector sees. In section 3 this modulation was shown to be a result of the satellite sheath geometry. Figure $3 a$ showed the detector A spin modulation for the same period as Figure $8 a$. Between 0800 and 1400 UT very low densities would be expected, because the spin count rate peak lies at spin phase angles where the count rates are reduced by the solar cell anomaly problem. Also, the automated first moment computation only looks at spin phase angles away from this region. Hence in Figure $8 a$ between $\sim 0800$ and $\sim 1400$ UT, reduced detector A densities would be expected and are in fact seen. The very erratic density variations between 1300 and 1700 UT are obtained because not all spin phase angles are sampled in a 6-min period, and if the relatively narrow spin phase peak were not sampled the resulting integration would produce a much reduced first moment (density). A dashed line from about 0600 to 1500 UT indicates a more probable variation for the fieldaligned density. The actual detector A density would be somewhat larger than those shown because the integration is over a relatively wide range of spin phase angles, much wider than the half width of the spin modulation peak. Correcting for this effect would be difficult, since a detailed quantitative understanding of the sheath would be required.

Since the detector A and B densities are quite different, the distribution function is nonisotropic; therefore the two densities only apply to those regions of the distribution function sampled by the detectors. Detector A samples pitch angles centered between $12^{\circ}$ and $25^{\circ}$. The center angle is modulated between these two values over the 24-hour orbital period. Over the 6-min integration period the detector A center pitch angle is constant. Detector B, on the other hand, covers pitch angles from $65^{\circ}$ to $115^{\circ}$ in a satellite spin period of $6 \mathrm{~s}$. The distribution function represented by Figure $3 a$ is highly field aligned, and consequently, the first moment of the cold part of the distribution function (density) cannot be derived from the partial phase space sampled by the SPA. This total proton density will be significantly lower than the A trace but probably slightly larger than the B trace in Figure $3 a$. From Figure $8 a$ it is evident that the degree of field alignment exceeds an order of magnitude between pitch angles of $10^{\circ}-20^{\circ}$ and $60^{\circ}-90^{\circ}$.
Figure 9 shows the refilling cold proton densities on June 15, 1980 (day 167), a slightly disturbed day with $\Sigma$ $K p=10^{-}$. The A detector density exceeds that of $\mathrm{B}$ by over an order of magnitude for most of the day with only a short period after 2100 UT, when the two densities ap. proach each other. During this latter period the detector A peak lies at spin phase angles associated with the solar cell anomaly (see Figure 4). Hence the detector A density probably varies more like the dashed line in Figure 9.

Both days 8 and 167 show field-aligned proton fluxes whose densities build up over a 24-hour period. These days had a daily $K p$ sum of 12 and $10^{-}$, respectively, still relatively undisturbed, and were chosen to show the fieldaligned nature of the detector A count rate during a 24. hour period. More disturbed days show all the above characteristics but in addition they are further complicated by the presence of a very sharp density drop in the 1600-2400 LT sector. At this point the GEOS 2 satellite would cross out of the 1 - to $10-\mathrm{cm}^{-3}$ density plasma which had built up, as in Figures $8 a$ and 9, into a region where both detectors $A$ and $B$ observed count rates at the noise level of $<0.1 \mathrm{~cm}^{-3}$. From these low densities the density would gradually increase as is shown between 0000 and 0600 UT in Figures $8 a$ and 9. The location of this discrete boundary as observed by the corotating geosynchronous satellite is dependent on the level of magnetic activity. Figure 10 shows the local time of this boundary as a function of the corresponding 3-hourly $K p$ index for 74 days between January 25, 1980, and May 31, 1980. All days for which data are available are plotted, although 15 very quiet days showed no cutoff boundary, since the satellite remained in the plasmasphere all day. This boundary also correlates with a sudden increase in the fluxes of hot electrons, the so-called injection boundary.

\subsection{Field-Aligned Proton \\ Pitch Angle Distribution}

In neither the summer nor winter satellite orientation is full pitch angle coverage obtained. For most of the time, detector $A$ views at a constant pitch angle $\delta_{2}$, while detector B scans pitch angles from $60^{\circ}$ to $110^{\circ}$ each spin. During flux tube refilling periods, about $85 \%$ of all GEOS 2 data, detector A count rate exceeds that of B by about a

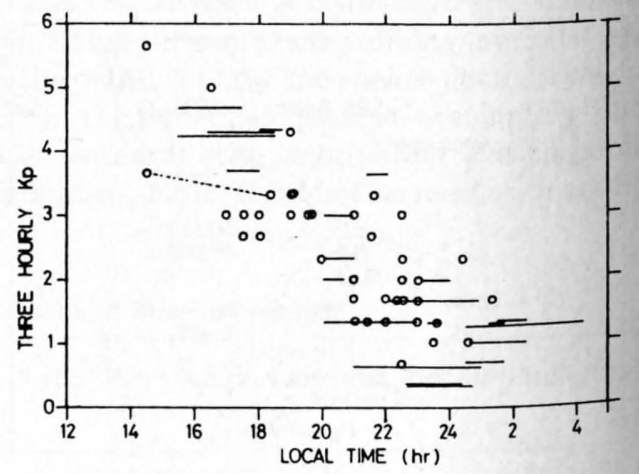

Fig. 10. Distribution of the cold proton cutoff boundary as a function of the 3-hourly $K p$ index. The circles indicate very well defined boundaries, while the lines indicate wider boundary. The dashed line indicates a day with two well-defined boundaries. 
factor of 10. Since detector B does not show a marked pitch angle modulation, the pitch angle distribution is field aligned with the bulk of the anisotropy occurring at pitch angles below $60^{\circ}$. At these low pitch angles the SPA only samples one angle. While the satellite is being inverted at equinox, the satellite spin axis is in the equatorial plane for a brief period. At this time the SPA B detector scans through almost all pitch angles. Unfortunately, the solar cell malfunction normally makes these data exceedingly difficult to reconstruct; however, for very brief periods (tens of minutes) the satellite was in eclipse and the solar cell anomaly disappeared. During these times complete pitch angle distributions were obtained.

Figure 11 shows one of the best field-aligned examples of the thermal proton pitch angle distribution taken during half-inverted eclipse conditions. These data were obtained on September 17, 1978 (day 258), at 2115-2130 UT. On this day the proton densities exceeded $15 \mathrm{~cm}^{-3}$, and the distribution was becoming isotropic. The field alignment shown in Figure 11 is quite mild, with only a factor of 3 between $0^{\circ}$ and $90^{\circ}$, in contrast to the order of magnitude difference between detector $\mathrm{A}$ and $\mathrm{B}$ under normal refilling. Since eclipse conditions are only met around local midnight, at a time when the flux tubes are at their most depleted due to storm/substorm activity, none of the other half-inverted eclipse data show stronger field alignment. Most of these other data have count rates which are near the noise level. Figure 11 does show that the field alignment occurs at low pitch angles. If we allow for the detector $\mathrm{B} 8^{\circ} \times 32^{\circ}$ aperture geometry, the field alignment would be increased significantly. A simple deconvolution, assuming the $\mathrm{B}$ detector's angular response was a narrow rectangle with a length of $32^{\circ}$, increases the field alignment by a factor of 3 . Even allowing for this correction the field alignment does not reach that observed under normal refilling conditions. Although the degree of field alignment is high, it is not a sharp source cone population. The source cone half angle at the geosynchronous location is typically less than $3^{\circ}$. Detector A observes large fluxes of protons at pitch angles between $12^{\circ}$ and $25^{\circ}$, given that its aperture is $\pm 9^{\circ}$. This would preclude direct observations in the loss cone.

\section{Discussion}

From the previous section it is evident that the sheath interaction with measurements of thermal protons cannot be ignored. However, because of the limited angular coverage of the data set, i.e., one pitch angle with a relatively wide angular width, a rigorous calculation cannot be made to deduce the details of the refilling proton fluxes. Qualitatively, several conclusions can be arrived at concerning the refilling observations. Over 2 years of observational data from GEOS 2 show proton fluxes similar to that shown in Figures $8 a$ and 9. This indicates that the refilling anisotropic plasma is present whenever the geosynchronous flux tube at $6.6 R_{E}$ has an equatorial density $\lesssim 10 \mathrm{~cm}^{-3}$. These refilling fluxes show no daynight diurnal trend, indicating that the degree of solar illumination in the ionosphere is relatively unimportant to the refilling process at the equator. The fluxes, or in-

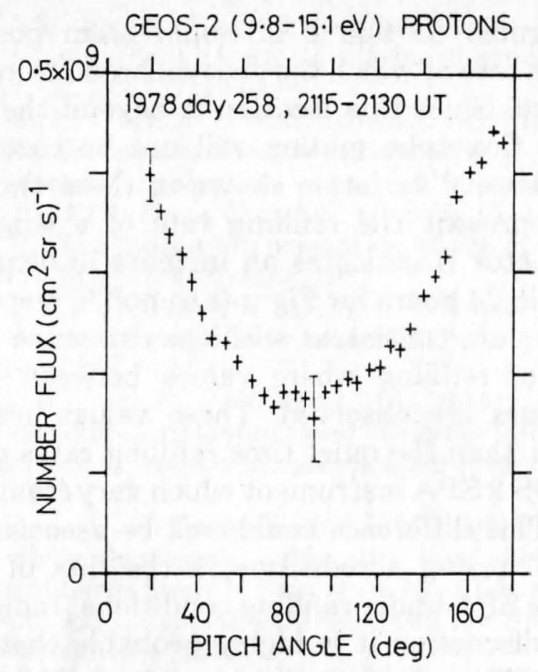

Fig. 11. Detector B number flux as a function of pitch angle during eclipse and half-inverted satellite mode on day 258, 1978.

ferred densities at low and high pitch angles of these refilling protons, appear to increase with time. It is difficult to prove this conclusively, since the GEOS 2 satellite is corotating and is in a region where flux tubes are probably not corotating. The fact remains that in the 1600-2400 LT range the satellite under active magnetospheric conditions would cross a sharp density discontinuity into a region of very low density. From this point onward the field-aligned proton fluxes would be observed, their fluxes increasing continually with time. This flux increase would continue until the next day when the satellite would again cross the dark sector boundary. On some days (very quiet days) no such subsequent boundary was present, and the fluxes would continue to increase until the density approached $\sim 20 \mathrm{~cm}^{-3}$ and the plasma would become isotropic and plasmaspheric in character. As the densities increased, the degree of field alignment deduced from the detector $\mathrm{A} / \mathrm{B}$ ratio would decrease. From the data it is not possible to infer whether the observed fieldaligned component is simply being swamped by an increasing isotropic component or whether the refilling plasma pitch angle distribution is indeed being modified.

The data taken during the half-inverted mode, i.e., Figure 11, and the inferences made in section 4.2 concerning the strong detector A spin modulation for pitch angles ranging from a few degrees to $30^{\circ}$ imply the pitch angle distribution is not limited to the $\sim 3^{\circ}$ ionospheric source cone. Indeed, the characteristic $1 / 2$ angle for the field-aligned component is probably between $10^{\circ}$ and $20^{\circ}$. Such an enhanced $1 / 2$ angle would seem to indicate that pitch angle scattering out of the source cone has occurred. The refilling progresses both day and night, indicating that the plasma source is above the ionosphere and therefore is likely to be consistent with these larger equatorial field-aligned angles. The $10^{\circ}$ and $20^{\circ}$ source angles would correspond to $90^{\circ}$ pitch angles at altitudes of 9000 and $17,000 \mathrm{~km}$, respectively. These altitudes are quite consistent with the exosphere as the source of the protons. Hence the field-aligned refilling signatures do not necessarily require pitch angle scattering or other complicated mechanisms to explain their shape. 
From Figures $8 a$ and 9 it would seem possible to estimate the rate at which the geosynchronous location is being refilled. Since this location is beyond the plasmasphere, the flux tube motion will not be corotational; hence the density variation shown in these two figures does not represent the refilling rate of a single "flux tube." Detector B indicates an increase in density of 1 and $3 \mathrm{~cm}^{-3}$ in 24 hours for Figures $8 a$ and 9 , respectively. These values are consistent with the extensive GEOS 2 data base of refilling where values between 1 and 5 $\mathrm{cm}^{-3 / 24}$ hours are observed. These values are significantly lower than the quiet time refilling rates observed by the GEOS 2 SPA instrument which vary from 10 to 40

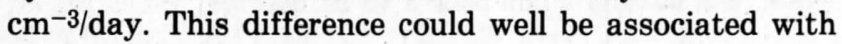
the sheath causing a reduction in the flux of protons reaching the SPA under refilling conditions. Indeed, from the earlier discussion it is highly probable that only at selected satellite spin phase angles and small pitch angles would an appreciable percentage of the magnetospheric flux reach the SPA. Consequently, it is likely that these refilling rates are lower limits and that quantitatively accurate densities will only be arrived at with a fuller quantitative treatment of the satellite sheath effects. These same limitations apply to the determination of the absolute field-aligned refilling proton fluxes. Once the density has become large $\left(>10 \mathrm{~cm}^{-3}\right)$, the plasma becomes isotropic, and the satellite potential decreases to $\sim \pm 0.5$, the problem of significant sheath masking goes away.

\section{Summary}

In this paper we have presented the general characteristics of the refilling cold plasma proton pitch angle distribution on flux tubes beyond the plasmasphere at the geomagnetic equator. Difficulties and uncertainties in interpreting these low-energy observations are partially overcome by being able to qualitatively identify the main source of these problems. These cold plasma observations complement the DE 1 lower-altitude observations of refilling plasma flows [Sojka et al., 1983] and the GEOS 1 observations of Wrenn et al. [1984]. The refilling plasma has the following characteristics:

1. The pitch angle distribution of $\lesssim 3-\mathrm{eV}$ protons is highly field aligned with half width angles between $10^{\circ}$ and $20^{\circ}$.

2. As the flux tube refills and the isotropic cold proton component increases, the field-aligned component appears to become less field aligned. Our observations are unable to resolve whether indeed the field-aligned component changes or whether it is simply being swamped by the increasing isotropic component.

3. The observations show no day-night refilling asymmetry. Refilling fluxes continually increase with abrupt density decreases in the $1600-2400$ LT sector which are associated with changes in magnetic storm activity in the magnetosphere. These decreases are predominantly found in the evening sector region and are strongly correlated with magnetic activity and lie just prior to the "injection boundary."

4. The observed pitch angle half widths of $10^{\circ}$ to $20^{\circ}$ would correspond to upper limits on the exospheric source regions of between 9000 and $17,000 \mathrm{~km}$ altitude if single particle kinetic motion is applied.
5. Qualitatively, the refilling rates and the fluxes of the refilling protons cannot be determined without a better quantitative understanding of the satellite sheath However, lower limits appear to be between 1 and 5 $\mathrm{cm}^{-3} /$ day at the equator for $L=6.6 R_{E}$.

In arriving at these plasma characteristics the study identified a significant sheath modification of the instrument response to field-aligned proton fluxes. Namely, the SPA detector, which was deliberately mounted to view antiparallel to the satellite spin axis to minimize spin modulation, observed exceedingly strong spin modulation. These modulations were always present in regions of refilling cold plasma. Even though detector A viewed at a constant pitch angle over a spin period, the modulation amplitude exceeded an order of magnitude. Over a 24hour period the modulation count rate peak and the mag. netic field showed strongly correlated spin phase-UT variations. On the basis of a numerical sheath model of the GEOS SPA boom configuration by the NASCAP model, a qualitative explanation for this effect was described.

At this time we do not claim to have a qualitative proof of the observed sheath-aperture spin asymmetry; indeed, with data from only one pitch angle it is improbable that a full sheath-plasma deconvolution is possible. However, we have identified and demonstrated a potentially very powerful technique to carry out in situ plasma sheath studies. This technique does not require any new technology. It would require a boom-mounted particle detector package which can be biased in relation to the satellite. Ideally, the distance of this package from the satellite would be variable. The particle detectors would have small angular acceptance angles and be able to scan azimuth angles as well as pitch angles for a given satellite spin phase orientation. Count rate distribution functions of cold ions and electrons obtained from such an instrument would provide the following unique observational possibilities:

1. By assuming a knowledge of the interaction process, the sheath properties could be mathematically deconvoluted from the plasma distribution function. Since we already think we know these processes, this would enable direct experimental testing.

2. Resulting from possibility 1 would be a plasma distribution function of significantly higher resolution than that which would be attained in any other way. This would enable cold plasma properties like Mach number, pitch angle anisotropy, and temperature anisotropy to be determined for the refilling cold proton fluxes. Current theoretical models already predict a wide range of values for these parameters but are unable to decide which are unphysical in magnetospheric contexts due to lack of observational boundary conditions.

3. By being able to map the satellite sheath in macroscopic detail, in situ studies of plasma sheaths and differential charging would become practical.

4. One particular aspect of major significance would be the ability to explore satellite wakes, a plasma region of importance not only for man-made satellites but also important in the wider field of plasma physics.

The above list of scientific topics are all under extensive theoretical study; however, the necessary complementary experimental studies are lagging behind primarily 
because of the historical difficulties in making these cold plasma-sheath measurements. Both the GEOS 1 and 2 satellites and more recently the P78-2 (SCATHA) satellites have carried instrumentation to study these problems and have made some progress, which indicates the above tasks are feasible. In addition, some work is being undertaken to actively control the satellite potential by using plasma emitters. This would enable a more direct method of attaining the object of possibility 2 . In this paper we feel that we have demonstrated the feasibility of carrying out such in situ measurements.

Acknowledgments. This research is part of the Mullard Space Science Laboratory program, directed by R. Boyd, and funded by a grant from the Science and Engineering Research Council (SERC) of the United Kingdom. Use of the SERC computing facilities at the Rutherford Appleton Laboratory is gratefully acknowledged. Very helpful discussions with A. D. Johnstone are acknowledged. A. Norris and R. Gowen are thanked for their assistance with data handling and programming.

The Editor thanks K. Knott and R. C. Olsen for their assistance in evaluating this paper.

\section{REFERENCES}

Decreau, P. M. E., J. Etcheto, K. Knott, A. Pedersen, G. L. Wrenn, and D. T. Young, Multi-experiment determination of plasma density and temperature, Space Sci. Rev., 22, 633-645, 1978.

Deforest, S. E., Electrostatic potentials developed by ATS-5, in Photon and Particle Interactions With Surfaces in Space, edited by R. J. L. Garard, pp. 263-276, D. Reidel, Dordrecht, Netherlands, 1973.

Deforest, S. E., and C. E. McIlwain, Plasma clouds in the magnetosphere, J. Geophys Res., 76, 3587-3611, 1971.

Garrett, H. B., The charging of spacecraft surfaces, Rev. Geophys. Space Sci., 19, 577-616, 1981.

Horwitz, J. L., Major questions on the interchange of thermal plasmas between the ionosphere and plasmasphere, J. Atmos. Terr. Phys., 45, 765-775, 1983.

Johnson, J. R. E., Magnetospheric studies with suprathermal plasma analysers on the ESA GEOS satellites, Ph.D. thesis, Univ. of London, London, 1981.

Kalweit, C., Report on the GEOS-2 power supply anomaly, Rep. Ref. $T F / C R / 6621$, Eur. Space Agency, Neuilly, France, and Eur. Space Res. and Technol., 1979.

Katz, I., J. J. Cassidy, M. J. Mandell, G. W. Schnuelle, P. G. Steen, and J. C. Roche, The capabilities of the NASA charging analyzer program, Spacecraft Charging Technology-1978, NASA CP-2071/AFGL-TR-79-0082, edited by R. C. Finke and C. A. Pike, 101-122, 1978.

Knott, K., P. M. E. Decreau, A. Korth, A. Pedersen, and G. L.
Wrenn, The potential of an electrostatically clean geostationary satellite and its use in plasma diagnostics, Planet. Space Sci., 32, 227-237, 1984.

Mandell, M. J., I. Katz, G. W. Schnuelle, P. G. Steen, and J. C. Roche, The decrease in effective currents due to saddle points in electrostatic potentials near differentially charged spacecraft, IEEE Trans. Nucl. Sci., 6(NS25), 1313-1321, 1978.

Olsen, R. C., The hidden ion population of the magnetosphere, J. Geophys. Res., 87, 3481-3488, 1982.

Olsen, R. C., C. E. McIlwain, and E. C. Whipple, Observations of differential charging effects on ATS 6, J. Geophys. Res., 86, 6809-6819, 1981.

Parker, L. W., and E. C. Whipple, Jr., Theory of spacecraft sheath structure, potential, and velocity effects on ion measurements by traps and mass spectrometers, J. Geophys. Res., 75, 4720-4733, 1970.

Sagalyn, R. C., D. E. Donatelli, and I. Michael, Proceedings of the Air Force Geophysics Laboratory Workshop on Natural Charging of Large Space Structures in Near Earth Polar Orbit: 14-15 Sept., 1982, Rep. AFGL-TR-83-0046, Air Force Geophys. Lab., Bedford, Mass., 1983.

Sojka, J. J., R. W. Schunk, J. F. E. Johnson, J. H. Waite, and C. R. Chappell, Characteristics of thermal and suprathermal ions associated with the dayside plasma trough as measured by the Dynamics Explorer retarding ion mass spectrometer, $J$. Geophys. Res., 88, 7895-7911, 1983.

Whipple, E. C., Jr., Theory of the spherically symmetric photoelectron sheath: A thick sheath approximation and comparison with the ATS- 6 observations of a potential barrier, $J$. Geophys. Res., 81, 601-607, 1976.

Whipple, E. C. Jr., Potentials of surfaces in space, Rep. Prog. Phys., 44, 1197-1225, 1981.

Wrenn, G. L. GEOS 2 in space collision?, Nature, 274, 631, 1978.

Wrenn, G. L., A. D. Johnstone, and J. F. E. Johnson, Spacecraft charging studies in Europe, Report MSSL-G795-01, MSSL, Holmbury St. Mary, Dorking, Surrey, England, 1979.

Wrenn, G. L., J. F. E. Johnson, and J. J. Sojka, The suprathermal plasma analysers on the ESA GEOS satellites, Space Sci., Instrum., 5, 271-293, 1981.

Wrenn, G. L., J. J. Sojka, and J. F. E. Johnson, Thermal protons in the morning magnetosphere: Filling and heating near the equatorial plasmapause, Planet. Space Sci., 32, 351-363, 1984.

J. F. E. Johnson, Department of Physics, University of Southampton, Southampton, S09 5NH, United Kingdom.

J. J. Sojka, Center for Atmospheric and Space Sciences, Utah State University, Logan, UT 84322.

G. L. Wrenn, Mullard Space Science Laboratory, Holmbury St. Mary, Dorking, Surrey, RH5 6NT England.

(Received March 23, 1984;

revised July 2, 1984; accepted July 3,1984 .) 